\title{
Developing Model Cathodes to Study Interfacial Ion Diffusion
}

\author{
Bilash KC ${ }^{1}$, Jacob R. Jokisaari ${ }^{1}$, Robert F. Klie ${ }^{1}$ \\ 1. Department of Physics, University of Illinois at Chicago, Chicago, IL, U.S.A
}

Li-ion batteries, the current paradigm of electrochemical energy storage technologies, face increasing demands for large-volume production of highly efficient and stable devices. The search for new cathode materials that can intercalate Li-ions more efficiently and at higher reversible concentrations is still a highly active research area, with considerable interest on lithium transition metal oxides, such as $\mathrm{LiMn}_{2} \mathrm{O}_{4}$. Studies have shown that thin layer of simple oxide/complex oxide coating of spinel $\mathrm{LiMn}_{2} \mathrm{O}_{4}$ allows improved electrochemical performance such as capacity and cyclability.[1,2] However, these materials are generally only available as powders, making detailed study of surfaces and interfaces difficult. To achieve such detailed characterization, a thin film model material system is highly desirable.

We used Molecular Beam Epitaxy (MBE) to grow $\mathrm{LiMn}_{2} \mathrm{O}_{4}$ single crystal thin films on $\mathrm{SrTiO}_{3}$ substrates as model cathodes. MBE thin film synthesis allows for atomically flat layers to be grown with well-defined surface terminations, orientations, defect concentrations and a homogenous distribution of compositional elements, which provides a good opportunity to characterize the cathodes in pristine and cycled states. The simplicity of single crystals compared to polycrystalline particulate cathode materials allows specific structural and chemical aspects of intercalation to be isolated and examined using nanoscale characterization techniques, such as transmission electron microscopy (TEM), scanning transmission electron microscopy (STEM), and electron energy loss spectroscopy (EELS), as well as more common Xray diffraction (XRD), or X-ray photoemission spectroscopy (XPS). A considerable body of experimental evidence has shown that crystallinity, compositional homogeneity, crystallographic orientation, point defects, and grain boundaries have an effect on Li ion transport. [3,4] Single-crystal model systems give access to each of these properties, and can be optimized for atomic-scale characterization, such as thinning to full electron transparency for STEM. Furthermore, these experiments will be essential for careful study of SEI layer, Li-ion diffusivity, and understanding structural framework changes in cycled cathodes to improve cyclability and capacity retention.

For initial characterization, several $\sim 100 \mathrm{~nm}$ thick films were grown in via MBE in an ultrahigh vacuum (UHV) environment. Elemental flux rate, oxygen partial pressure, and growth temperature were adjusted to obtain higher crystalline films. Structural and chemical characterization was carried out using aberration-corrected STEM, energy-dispersive X-ray spectroscopy (EDS), EELS, as well as XRD and XPS for comparison to conventional electrochemical systems. Figure 1(a-e) shows the XRD pattern of film, XPS spectrum of Mn L-edge, EDS quantitative analysis, and Li-EEL spectrum for one of the samples. Figure 2(a-c) shows the thickness, crystallinity, defects, and diffraction spots from the same film obtained using a JEOL 3010 TEM. The images show we have successfully synthesized MBE thin films of lithium manganate suitable both for electrochemical testing and for high-resolution aberration-corrected STEM/EDS/EELS characterization.

References:

[1] J. Zhao J \& Y. Wang, J. Phys. Chem. C 116 (2012), p. 11867-11876.

[2] J. Li et.al, ACS Appl. Mater. Interfaces 6 (2014), p. 18742-18750

[3] J. S. Kim et. al, Nano Lett. 12 (2012), p. 6358-6365. 
[4] R. Yazami in "Nanomaterials for Lithium-Ion Batteries" ed. R.Yazami (CRC press, Florida) p.261,269,277.

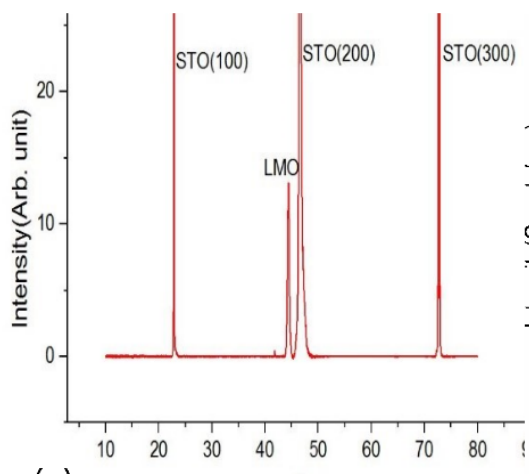

(a)
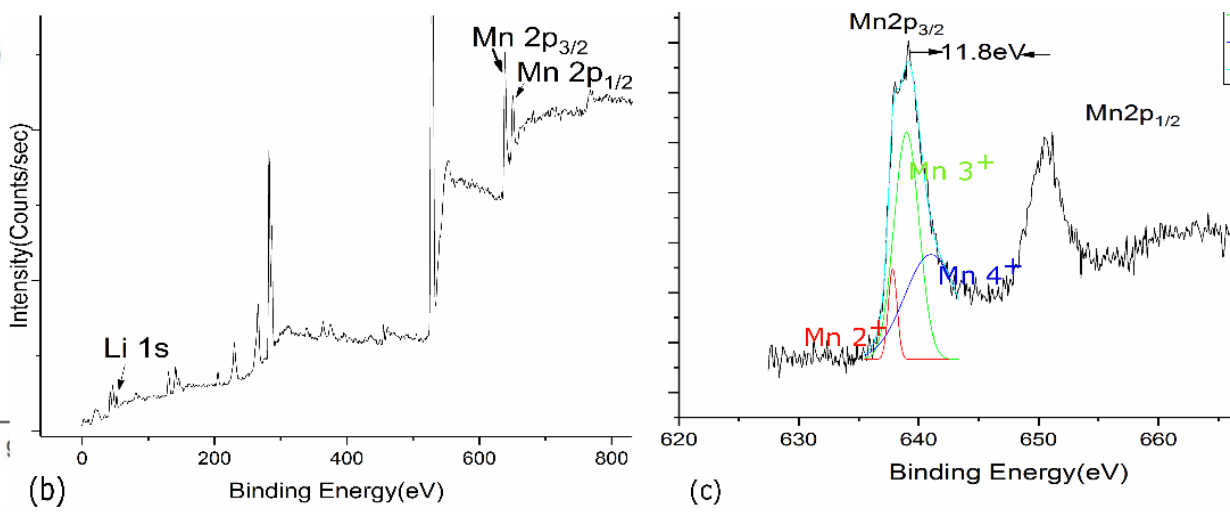

(c)
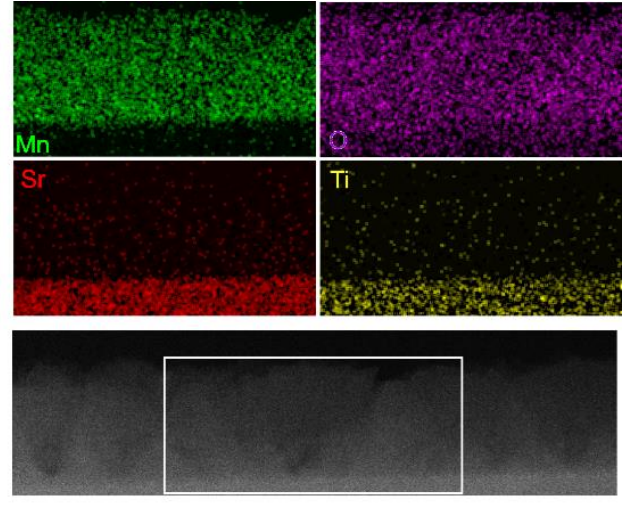

Figure 1. (a) XRD pattern, (b) XPS spectrum of Li $K$-edge, Mn $L$-edge, (c) Mn $L$-edge, (d) EDS spectrum, (e) Li-EELS spectrum taken from the $\mathrm{LiMn}_{2} \mathrm{O}_{4}$ epitaxial thin films.

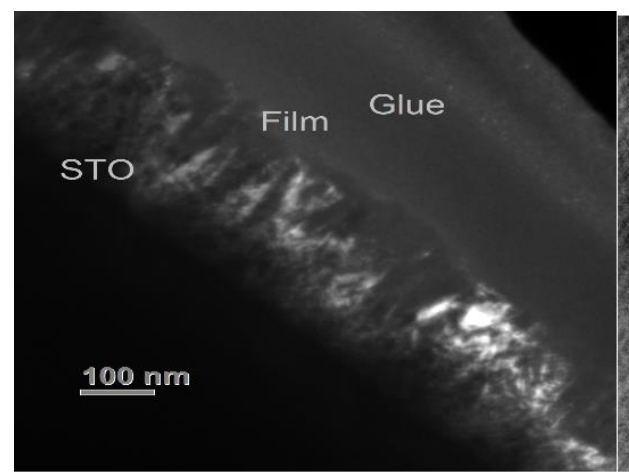

(a)

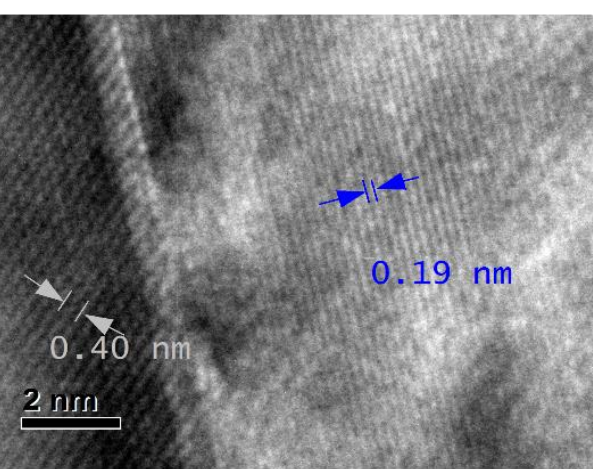

(b)

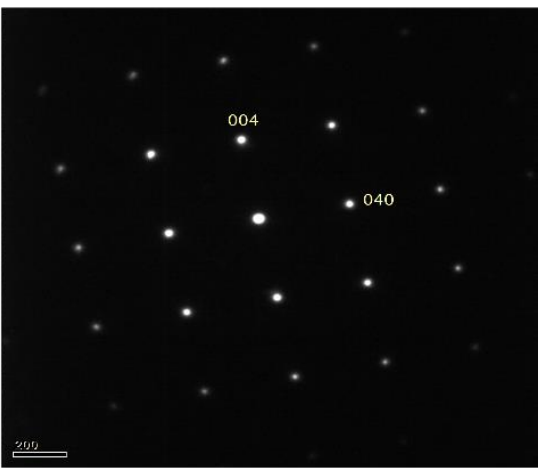

(c)

Figure 2. TEM images and electron diffraction pattern showing (a) $\mathrm{LiMn}_{2} \mathrm{O}_{4}$ film thickness, (b) thin film lattice fringes, (c) Selected Area Diffraction image 